\title{
Translocation der(13;21)(q10;q10) in Skeletal and Extraskeletal Mesenchymal Chondrosarcoma
}

\author{
Sabine Naumann, Ph.D., Pamela A. Krallman, B.A., K. Krishnan Unni, M.D., Mary E. Fidler, M.D., \\ James R. Neff, M.D., Julia A. Bridge, M.D. \\ Departments of Pathology and Microbiology (SN, PK, JRN, JAB), Orthopaedic Surgery (JRN, JAB), and \\ Pediatrics (JAB), University of Nebraska Medical Center, Omaha, Nebraska; and Department of Laboratory \\ Medicine and Pathology (KKU, MEF), Mayo Clinic, Rochester, Minnesota
}

\begin{abstract}
Cytogenetic studies of mesenchymal chondrosarcoma are few and to date, no specific or recurrent aberrations have been found. In this investigation, the cytogenetic and molecular cytogenetic (spectral karyotypic and fluorescence in situ hybridization) findings for two mesenchymal chondrosarcomas, one arising skeletally and the other extraskeletally, are reported. An identical Robertsonian translocation involving chromosomes 13 and 21 [der(13; 21)(q10;q10)] was detected in both cases, possibly representing a characteristic rearrangement for this histopathologic entity. Both cases also exhibited loss of all or a portion of chromosomes 8 and 20 and gain of all or a portion of chromosome 12. The observation of similar chromosomal abnormalities in both skeletal and extraskeletal mesenchymal chondrosarcoma supports a genetic as well as histopathologic relationship between these anatomically distinct neoplasms.
\end{abstract}

KEY WORDS: Cytogenetics, Fluorescence in situ hybridization, Mesenchymal chondrosarcoma, Spectral karyotype.

Mod Pathol 2002;15(5):572-576

Mesenchymal chondrosarcoma is a rare, malignant neoplasm that represents less than $2 \%$ of all chondrosarcomas (1). It occurs most frequently in young adults during the second or third decades of life and affects males and females nearly equally. Mesenchymal chondrosarcoma has a predilection for the craniofacial bones; however, the vertebrae, ribs, pelvis, and humerus are also frequent sites of in-

Copyright (C) 2002 by The United States and Canadian Academy of Pathology, Inc.

VOL. 15, NO. 5, P. 572, 2002 Printed in the U.S.A.

Date of acceptance: January 9, 2002

This work was supported in part by NIH/NCI P30CA36727, Nebraska Department of Health LB595, the John A. Wiebe, Jr. Children's Healthcare Fund, and the Scott Carter Foundation.

Address reprint requests to: Julia A. Bridge, M.D., Department of Pathology and Microbiology, 983135 Nebraska Medical Center, Omaha, NE 68198-3135; e-mail: jbridge@unmc.edu; fax: 402-559-6018. volvement. This neoplasm may also arise in extraskeletal locations. Histopathologically, mesenchymal chondrosarcoma typically exhibits a bimorphic pattern featuring solid, highly cellular areas of round or slightly spindled cells and islands of hyaline cartilage. The noncartilaginous elements often predominate, and such lesions can be confused histologically with Ewing's sarcoma or hemangiopericytoma (2).

Cytogenetic studies of mesenchymal chondrosarcoma are limited (3-7). In this study, three mesenchymal chondrosarcoma specimens from two patients subjected to cytogenetic and molecular cytogenetic analyses revealed a 13;21 Robertsonian translocation in all three samples as well as loss of chromosomes 8 and 20 material and gain of chromosome 12 material.

\section{MATERIALS AND METHODS}

Case Histories

\section{Case 1}

A 24-year-old woman presented to her local physician with a painless left lower quadrant abdominal mass in 1993. A needle biopsy revealed a malignant cartilaginous neoplasm consistent with mesenchymal chondrosarcoma. She subsequently received chemotherapy, followed by a resection of the retroperitoneal mass measuring $16 \times 10 \times 4$ $\mathrm{cm}$. Microscopically, the lesion was composed of solid sheets of primitive round cells and foci of mature cartilage with a relatively well-differentiated appearance (Fig. 1). Less than $10 \%$ necrosis could be appreciated in her surgically removed tumor.

Approximately 1 year after removal of her primary neoplasm, the patient developed a metastasis to her right kidney measuring $13 \times 11.5 \times 10.5 \mathrm{~cm}$. Subsequently she developed additional metastases, including multiple neck and lung metastases in 1996, rib metastases in 1997, humerus and pancreas metastases in 1999, and spine metastases in 


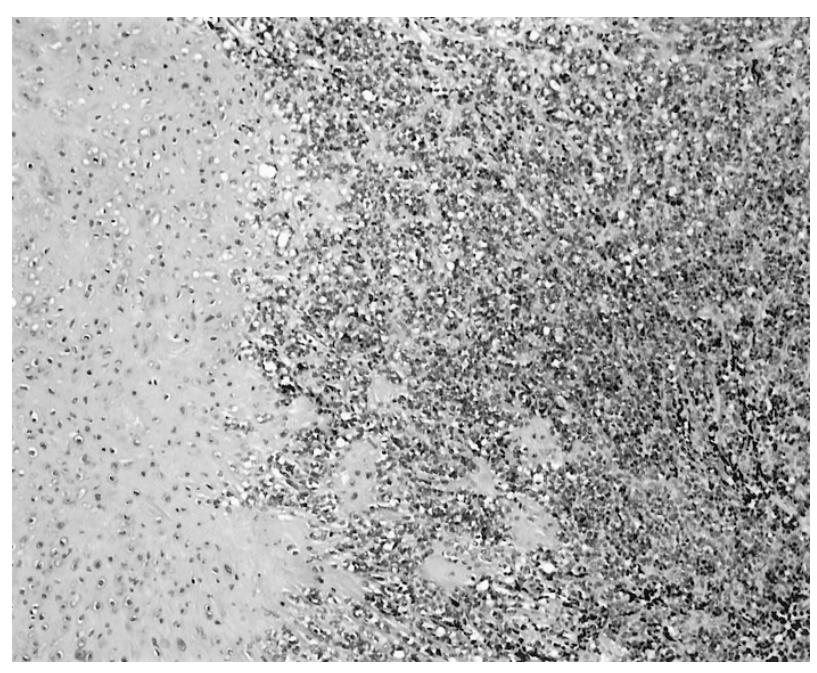

FIGURE 1. Case 1 mesenchymal chondrosarcoma with a richly cellular field of round tumor cells and an island of well-differentiated cartilage (hematoxylin-eosin stain $\times 160$ ).

2000. A representative portion of one of the rib metastases was submitted for cytogenetic analysis. Currently, the patient is alive with disease and undergoing radiation therapy.

\section{Case 2}

A 32-year-old man developed a mass at the junction of the middle and proximal one-third of the right femur in 1998. An open biopsy was interpreted as mesenchymal chondrosarcoma. A radical resection of the lesion was performed removing the mass with a segment of the shaft of the femur. The surgical defect was reconstructed using a deep frozen allograft with an interlocking intramedullary nail. The allograft/host junctures healed without complication. The histologic appearance of the resection specimen was similar to that described for Case 1.

Twenty-eight months later the patient developed a tender soft tissue nodule in the right deltopectoral groove as well as another tender nodule involving the right temporalis muscle. A bone scan revealed intense uptake in both regions. Both lesions were removed with material preserved for routine histopathology and cytogenetics. The pathologic diagnosis confirmed metastatic mesenchymal chondrosarcoma at both sites. The patient is currently alive with disease.

\section{Cytogenetic Analysis}

A representative sample of the rib metastasis of Case 1, and representative samples of the metastatic lesions involving the deltoid and temporalis muscles of Case 2, were submitted for cytogenetic analysis. In situ culturing, harvesting and preparation of slides were performed as previously described (8). Chromosome analysis was performed on GTG-banded (Giemsa/trypsin) metaphases and the karyotypes were expressed according to international guidelines (9).

\section{Spectral Karyotypic Analysis (SKY)}

Spectral karyotyping was performed on an unstained cytogenetic preparation of the deltoid muscle specimen of Case 2 according to the manufacturer's instructions (Applied Spectral Imaging, Carlsbad, CA). Ten metaphase cells were analyzed. Spectral images were acquired and analyzed with a SD 200 spectral bio-imaging system (ASI Ltd., Migdal Haemek, Israel) attached to a Zeiss microscope (Axioskop 2). The generation of a spectral image was achieved by acquiring approximately 100 frames of the same image that differ from each other only in the optical path difference (OPD). The images were stored in a computer for further analysis using the SKYVIEW software (version 1.6.1, ASI, Carlsbad, CA). Repeated efforts to obtain SKY results from 3-year-old destained cytogenetic preparations of Case 1 were unsuccessful (material was limited and no unstained preparations were available).

\section{Fluorescence In Situ Hybridization (FISH)}

A series of FISH experiments were performed on the same slide preparation utilized for SKY analysis of Case 2 after destaining. Specifically, bicolor FISH studies were performed using a biotin labeled coatasome 20 probe (Oncor, Gaithersburg, MD) in three separate experiments with spectrum green labeled whole chromosome painting probes 3, 8, and 11 (Vysis, Downers Grove, IL). Before each hybridization, the slide was washed at room temperature in $4 \mathrm{XSSC} / 0.1 \% \mathrm{NP} 40$ for 10 minutes and in 2XSSC for 5 minutes. After application of each probe mixture to the slide, a 1 minute codenaturation at $75^{\circ} \mathrm{C}$ and overnight hybridization at $37^{\circ} \mathrm{C}$ using the HYBrite ${ }^{\mathrm{TM}}$ system was performed (Vysis, Downers Grove, IL). Detection of signals was performed as previously described (10).

Images were prepared utilizing the Applied Image Analysis System (Applied Imaging, Pittsburgh, PA). As a quality control, metaphase cells prepared from normal peripheral blood lymphocytes were simultaneously hybridized with these probe combinations.

\section{RESULTS}

\section{Case 1}

Fourteen GTG-banded metaphase cells analyzed from the metastatic lesion of the spine revealed the following chromosomal abnormalities: 46,XX,del(8) (q24),+12, der(13;21)(q10;q10), $\operatorname{add}(20)(\mathrm{q} 13)[10] /$ 
92,idemx2[4] (Fig. 2). Four cells were karyotypically normal.

\section{Case 2}

Cytogenetic analysis of the temporalis muscle lesion and the deltoid muscle lesion revealed similar abnormal complements: 45,XY, $\operatorname{del}(4)(q 12 q 21)$,8, $\operatorname{del}(9)(\mathrm{p} 13),+\operatorname{del}(12)(\mathrm{q} 22), \operatorname{der}(13 ; 21)(\mathrm{q} 10 ; \mathrm{q} 10)$, $\operatorname{add}(18)(\mathrm{p} 11.3),-20,+\operatorname{mar}[2] / 45$,idem, add (7) (q36) [3]/90,idemx2 [5]/90,idemx2, add(7)(q36)x2 [1], and 45,XY,del(4) (q12q21),-8, del(9) (p13), +del(12) (q22), $\operatorname{der}(13 ; 21)(q 10 ; q 10), \operatorname{add}(18)(p 11.3),-20,+\operatorname{mar}[3] /$ 45,idem,add(7)(q36) [1]/90,idemx2[4], respectively (Fig. 3).

SKY and FISH analyses confirmed the presence of the 13;21 translocation and revealed that the additional material located on the short arm of chromosome 18 was derived from chromosome 8 [der(18)t(8;18)(q21;p11.3)] and the marker chromosome could be defined as $\operatorname{der}(8) \mathrm{t}(8 ; 11)(\mathrm{p}$ ?; $) \mathrm{t}(8$; 20) (q?;?) (Fig. 4).

\section{DISCUSSION}

Translocations, or exchange of chromosomal material between two or more homologous chromosomes, are frequently encountered as tumor-specific anomalies in mesenchymal neoplasms (11). These translocations are often present as the sole cytogenetic abnormality and are therefore likely to be etiologic. At the molecular level, these chromosomal translocations produce highly specific gene fusions, most representing chimeric transcription factors (or co-factors). Only one chondrosarcoma subtype, extraskeletal myxoid chondrosarcoma, is reportedly characterized by recurrent or specific chromosomal translocations. These translocations, the $\mathrm{t}(9 ; 22)(\mathrm{q} 22$; $\mathrm{q} 12)$ or its variant, $\mathrm{t}(9 ; 17)(\mathrm{q} 22 ; \mathrm{q} 11.2)$, result in the fusion of the CHN (TEC) gene at 9q22 with the EWS gene at $22 \mathrm{q} 12$ or the TAF2N (RBP56) gene at $17 \mathrm{q} 11.2$ (12-18).

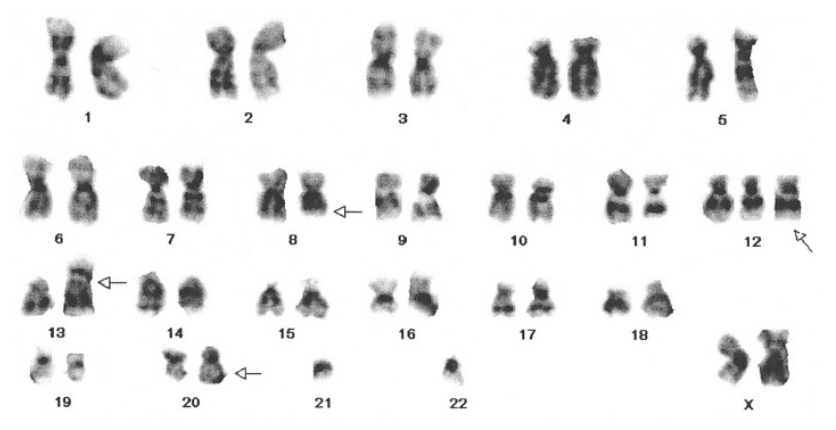

FIGURE 2. Representative G-banded karyotype of Case 1 exhibiting the following abnormal complement: 46,XX,del(8)(q24),+12,der(13;21) (q10;q10), add(20)(q13).
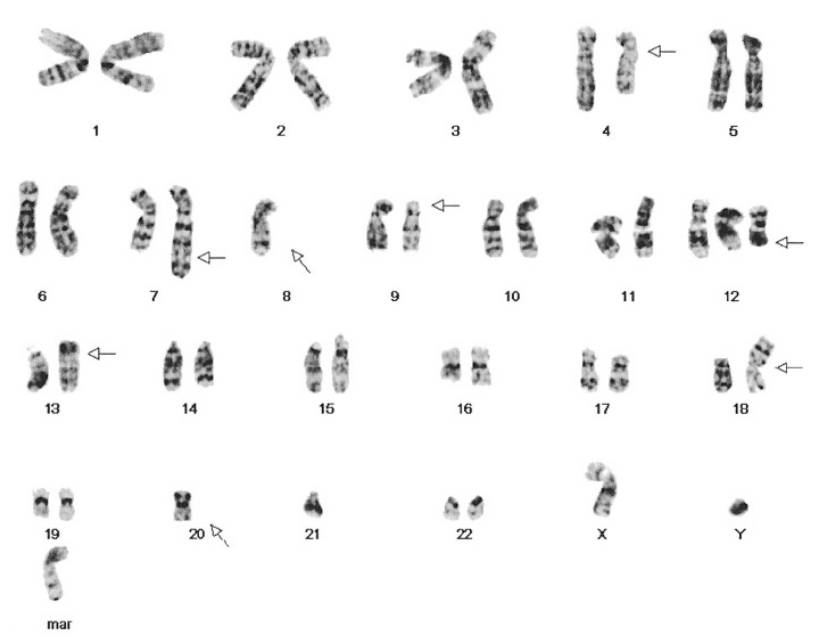

FIGURE 3. Representative G-banded karyotype of Case 2 exhibiting the following abnormal complement: 45,XY,del(4)(q12q21),add(7)(q36),-8,del (9) (p13), +del(12)(q22), der(13;21)(q10;q10), add(18)(p11.3),-20,+ mar.
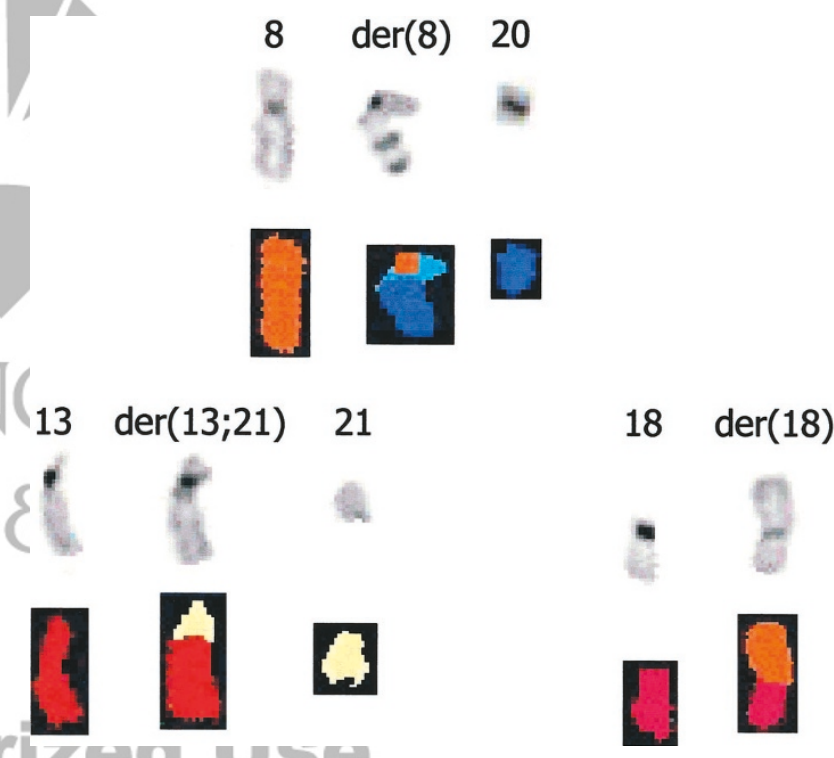

FIGURE 4. Partial SKY karyotype of Case 2 illustrating the origin of the additional material on the short arm of chromosome 18 [der(18)t(8;18) (q21;p11.3)] and the marker chromosome $[\operatorname{der}(8) \mathrm{t}(8 ; 11)(\mathrm{p} ? ;) \mathrm{t}(8 ; 20)(\mathrm{q} ? ; ?)]$, and confirming the presence of $a \operatorname{der}(13 ; 21)(\mathrm{q} 10 ; \mathrm{q} 10)$.

In this study, cytogenetic analysis of three mesenchymal chondrosarcoma specimens from two patients revealed an identical chromosomal translocation in all three specimens. This translocation, der(13;21) (q10;q10), differs from those typically associated with mesenchymal neoplasms as it is a Robertsonian translocation, a translocation that originates through centric fusion of the long arms of acrocentric chromosomes with simultaneous loss of both short arms. It is not clear what kind of molecular impact this rearrangement of centromeric regions may have in mesenchymal chondrosarcoma. The der(13;21)(q10;q10) translocation has not been detected in the five mesenchymal chon- 
drosarcomas that have been cytogenetically analyzed in prior studies (3-7).

Prior cytogenetic descriptions of mesenchymal chondrosarcoma have revealed diverse karyotypic findings (Table 1). The modal chromosomal number for two cases encompassed more than one ploidy level and ranged from 95 to 112 chromosomes in a mesenchymal chondrosarcoma arising in an 80-year-old man and 38 to 170 chromosomes in a strongly desmin immunoreactive mesenchymal chondrosarcoma arising in a 2-year-old boy (3, 5). These findings are in contrast to near-diploid complements observed in the current three specimens as well as three previously reported cases (4, $6,7)$. Interestingly, one case has been described as exhibiting the $t(11 ; 22)(q 24 ; q 12)$ characteristic of Ewing's sarcoma and peripheral primitive neuroectodermal tumors (pPNET) leading to the hypothesis that mesenchymal chondrosarcoma may be related to these neuroectodermal tumors (4). Unfortunately, studies were not performed to determine whether this case was also similar at the molecular level, i.e., featuring an EWS/FLI-1 fusion transcript.

In addition to sharing a der(13;21), the three specimens of the present study also featured loss of chromosomes 8 and 20 material and gain of chromosome 12 material. Loss of chromosome 8 material, particularly $8 \mathrm{q} 24.1$, the locus of the hereditary multiple exostoses gene EXT1, is common in both sporadic and hereditary osteochondromas and peripheral chondrosarcomas $(19,20)$. Disruption or rearrangement of the $20 \mathrm{ql} 3$ breakpoint was observed in the first case of the present series [add(20)(q13)] and possibly the second case. SKY and subsequent FISH studies with a chromosome 20 paint probe of the second case revealed that the marker chromosome of Case 2 was composed in part of chromosome $20 \quad[\operatorname{der}(8) \mathrm{t}(8 ; 11)(\mathrm{p}$ ?;?)t(8; 20) (q?;?)]. Rearrangement of $20 q 13$ was also seen in the mesenchymal chondrosarcoma cases reported by Mandahl et al. (3) and Szymanska et al. (7). Extra copies of all or a portion of chromosome 12 have been detected in five mesenchymal chondrosarcoma specimens including the three of the current series $(3,5)$. Aberrations of chromosome 12 have been described as common in conventional chondrosarcoma as well (21).

Marker chromosomes, structurally abnormal chromosomes in which no parts can be identified, were observed in three of the previously reported mesenchymal chondrosarcoma cases $(3,5,7)$. Molecular cytogenetic techniques to include SKY and FISH are of great value in defining the origin of marker chromosomes. In the current study, these methods were utilized to further define the additional material located on the short arm of chromosome 18 and a marker chromosome observed in Case 2. It is interesting to speculate as to whether

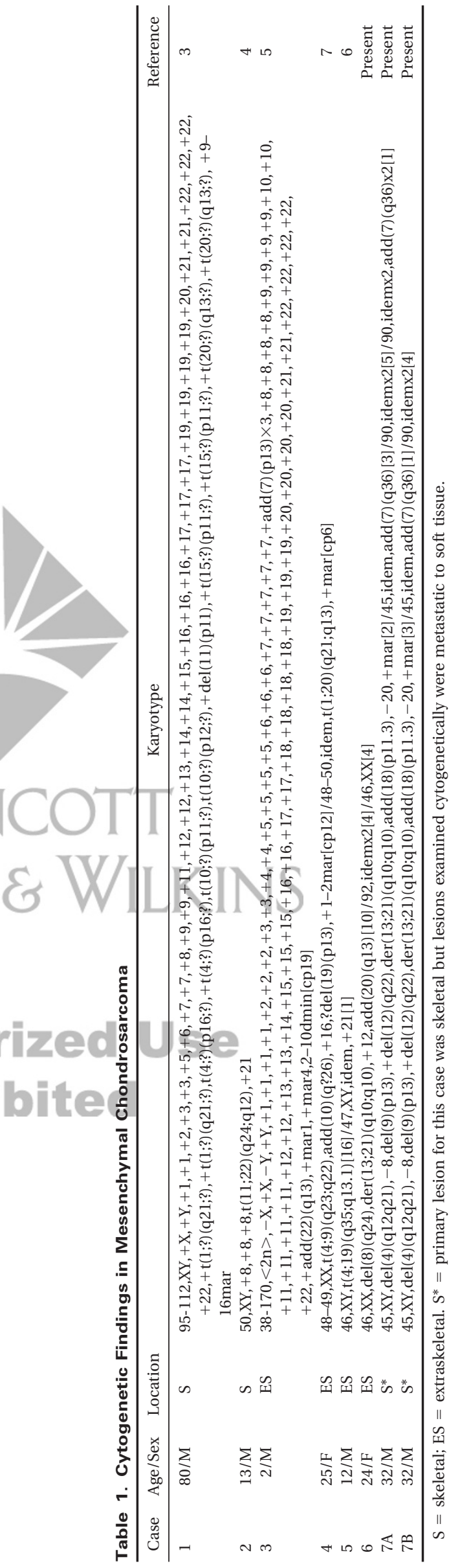


additional shared anomalies such as the der(13; 21) $(\mathrm{q} 10 ; \mathrm{q} 10)$ translocation or a variant of this translocation might be uncovered within the marker chromosomes of the previously reported cases if these approaches were used.

In summary, cytogenetic and molecular cytogenetic analyses of three mesenchymal chondrosarcoma specimens from two separate patients revealed a 13;21 Robertsonian translocation, loss of chromosomes 8 and 20 material and gain of chromosome 12 material in all three specimens. Identification of the $\operatorname{der}(13 ; 21)(\mathrm{q} 10 ; \mathrm{q} 10)$ translocation may be useful diagnostically for mesenchymal chondrosarcoma. Should additional studies confirm these data, this could lead to the identification of the gene(s) central to this neoplastic process.

Acknowledgments: The authors would like to thank Kimberly Christian for her expert secretarial assistance and Marilu Nelson, Patty Cattano, Jane Bayani, Warren Sanger, and Jeremy Squire for their valuable technical assistance and advice.
8. Safar A, Nelson M, Neff JR, Maale GE, Bayani J, Squire J, et al. Recurrent anomalies of $6 \mathrm{q} 25$ in chondromyxoid fibroma. Hum Pathol 2000;31:306-11.

9. Mitelman F (editor). ISCN (1995): an international system for human cytogenetic nomenclature. Basel: Karger; 1995.

10. Bridge JA, Fidler ME, Neff JR, Degenhardt J, Wang M, Walker C, et al. Adamantinoma-like Ewing's sarcoma: genomic confirmation, phenotypic drift. Am J Surg Pathol 1999;23:15965.

11. Bridge JA, Sandberg AA. Cytogenetic and molecular genetic techniques as adjunctive approaches in the diagnosis of bone and soft tissue tumors. Skeletal Radiol 2000;29:249-58.

12. Hinrichs SH, Jaramillo MA, Gumerlock PH, Gardner MB, Lewis JP, Freeman AE. Myxoid chondrosarcoma with a translocation involving chromosomes 9 and 22. Cancer Genet Cytogenet 1985;14:219-26.

13. Labelle Y, Zucman J, Stenman G, Kindblom LG, Knight J, Turc-Carel C, et al. Oncogenic conversion of a novel orphan nuclear receptor by chromosome translocation. Hum Mol Genet 1995;4:2219-26.

14. Clark J, Benjamin H, Gill S, Sidhar S, Goodwin G, Crew J, et al. Fusion of the EWS gene to CHN, a member of the steroid/ thyroid receptor gene superfamily, in a human myxoid chondrosarcoma. Oncogene 1996;12:229-35.

15. Brody RI, Ueda T, Hamelin A, Jhanwar SC, Bridge JA, Healey $\mathrm{JH}$, et al. Molecular analysis of the fusion of EWS to an orphan nuclear receptor gene in extraskeletal myxoid chondrosarcoma. Am J Pathol 1997;150:1049-58.

16. Antonescu CR, Argani P, Erlandson RA, Healey JH, Ladanyi M, Huvos AG. Skeletal and extraskeletal myxoid chondrosarcoma: a comparative clinicopathologic, ultrastructural, and molecular study. Cancer 1998;83:1504-21.

17. Panagopoulos I, Mencinger M, Dietrich CU, Bjerkehagen B, Saeter G, Mertens F, et al. Fusion of the RBP56 and CHN genes in extraskeletal myxoid chondrosarcomas with translocation $\mathrm{t}(9 ; 17)$ (q22;q11). Oncogene 1999;18:7594-8.

18. Sjogren H, Meis-Kindblom J, Kindblom LG, Aman P, Stenman G. Fusion of the EWS-related gene TAF2N to TEC in extraskeletal myxoid chondrosarcoma. Cancer Res 1999;59: 5064-7.

19. Bridge JA, Nelson M, Orndal C, Bhatia P, Neff JR. Clonal karyotypic abnormalities of the hereditary multiple exostoses chromosomal loci 8q24.1 (EXT1) and 11p11-12 (EXT2) in patients with sporadic and hereditary osteochondromas. Cancer 1998;82:1657-63.

20. Bovee JV, van Royen M, Bardoel AF, Rosenberg C, Cornelisse CJ, Cleton-Jansen AM, et al. Near-haploidy and subsequent polyploidization characterize the progression of peripheral chondrosarcoma. Am J Pathol 2000;157:1587-95.

21. Bridge JA, Bhatia PS, Anderson JR, Neff JR. Biologic and clinical significance of cytogenetic and molecular cytogenetic abnormalities in benign and malignant cartilaginous lesions. Cancer Genet Cytogenet 1993;69:79-90. 